\title{
The FANTASTIC FOUR proteins influence shoot meristem size in Arabidopsis thaliana
}

Vanessa Wahl ${ }^{1,3}$, Luise H Brand ${ }^{2,3}$, Ya-Long Guo ${ }^{3}$, Markus Schmid ${ }^{3 *}$

\begin{abstract}
Background: Throughout their lives plants produce new organs from groups of pluripotent cells called meristems, located at the tips of the shoot and the root. The size of the shoot meristem is tightly controlled by a feedback loop, which involves the homeodomain transcription factor WUSCHEL (WUS) and the CLAVATA (CLV) proteins. This regulatory circuit is further fine-tuned by morphogenic signals such as hormones and sugars.

Results: Here we show that a family of four plant-specific proteins, encoded by the FANTASTIC FOUR (FAF) genes, has the potential to regulate shoot meristem size in Arabidopsis thaliana. FAF2 and FAF4 are expressed in the centre of the shoot meristem, overlapping with the site of WUS expression. Consistent with a regulatory interaction between the FAF gene family and WUS, our experiments indicate that the FAFs can repress WUS, which ultimately leads to an arrest of meristem activity in FAF overexpressing lines. The finding that meristematic expression of FAF2 and FAF4 is under negative control by CLV3 further supports the hypothesis that the FAFs are modulators of the genetic circuit that regulates the meristem.

Conclusion: This study reports the initial characterization of the Arabidopsis thaliana FAF gene family. Our data indicate that the FAF genes form a plant specific gene family, the members of which have the potential to regulate the size of the shoot meristem by modulating the CLV3-WUS feedback loop.
\end{abstract}

\section{Background}

In contrast to animals, plant development is highly plastic, with new organs being formed continuously from pools of stem cells maintained in structures called meristems. This plasticity allows plants, within certain limits, to adapt their body shape in response to developmental, physical and environmental cues. The ability to form new organs throughout their life cycle requires tight control of the meristems to avoid unregulated growth. Plants have evolved an elaborate genetic network that controls meristem size and maintenance [1,2]. At the core of the network that regulates the size of the stem cell population in the shoot meristem are the homeodomain transcription factor WUSCHEL (WUS) and the CLAVATA (CLV) ligand-receptor system [1,3-5]. WUS is expressed in the organizing centre $(\mathrm{OC})$ of the meristem and positively regulates $C L V 3$ expression in the stem cells, which are localized above the OC [6]. CLV3 encodes a small

\footnotetext{
* Correspondence: Markus.Schmid@tuebingen.mpg.de

${ }^{3}$ Department of Molecular Biology, AG Schmid, Max Planck Institute for Developmental Biology, D-72076 Tübingen, Germany

Full list of author information is available at the end of the article
}

secreted peptide, which cell non-autonomously represses WUS in the OC [6-10]. It has recently been shown, that CLV3 directly binds to the ectodomain of the LRR receptor kinase CLV1 [11]. Similarly, it has been suggested that the receptor-like protein CLV2 interacts with the novel receptor kinase CORYNE (CRN; SUPPRESSOR OF OVEREXPRESSION OF LLP1-2, SOL2) to establish a functional CLV3 receptor [12,13]. Thus a feedback loop is established, which is essential to set up and maintain the stem cell population at the shoot meristem. However, the relationship between WUS and CLV3 is not static; the WUS-CLV system can compensate for changes in CLV3 expression over a wide range [14].

WUS expression is also controlled by phytohormones, which have been implicated in maintaining the stem cell system as well as setting up developmental compartments at the shoot meristem and in establishing the developmental fate of cells that are derived from the stem cell pool [reviewed in 2]. Besides hormones, sugars also appear to play an important role in establishing and maintaining meristem identity [reviewed in 15]. For example, it has been shown that growth arrest caused 
by loss of the WUS-related homeodomain factor STIMPY/WOX9 was rescued to a large extent by providing sucrose in the growth medium. This demonstrates that sucrose can compensate for the loss of at least some genes normally required for meristem development [16].

Here we present an initial characterization of a plantspecific gene family - FANTASTIC FOUR (FAF) - with four members in Arabidopsis thaliana (FAF1 - FAF4). We show that the $F A F$ genes are expressed throughout the life cycle of the plant, but exhibit strong temporal and spatial regulation. FAF2 and FAF4 expression was detected in the centre of the shoot meristem by RNA in situ hybridization and GUS reporter constructs. In addition, expression of the $F A F$ genes was detectable in the developing and mature vasculature. FAF gene overexpression negatively affected growth of both the shoot and the root. At the molecular level, the arrest of shoot growth was accompanied by a marked decrease in WUS expression. We further show that meristematic expression of FAF2 and FAF4 is under negative control by CLV3. Together these data suggest that the FAF proteins are capable of modulating shoot growth by repressing WUS in the OC of the shoot meristem.

\section{Results}

\section{The FANTASTIC FOUR (FAF) genes define a plant specific} gene family

The Arabidopsis thaliana FAF genes first caught our attention because two of them, FAF1 (At4g02810) and FAF2 (At1g03170), responded strongly and rapidly to a shift in photoperiod in a microarray experiment (Additional File 1 Figure S1) [17]. FAF1 and FAF2 belong to an uncharacterized gene family that also includes FAF3 and FAF4 (At5g19260, At3g06020, Table 1). Both pairs of genes, Arabidopsis thaliana FAF1/FAF2 and FAF3/ $F A F 4$, appear to be recently duplicated paralogs [18]. The proteins encoded by the $F A F$ genes do not contain any domains of known function (Table 1). In addition, the Arabidopsis thaliana genome encodes a more distantly related protein (At5g22090), which we call FAF-like (Additional File 1 Table S1). FAF and FAF-like proteins share several conserved domains, among them a stretch of acidic residues in their $\mathrm{C}$-terminal half.
Since the FAF genes have not been previously described, we wished to determine how widespread they are among other species. To address this question we searched publicly available sequence databases by reciprocal BLAST analysis for potential orthologs of the $F A F$ genes. Phylogenetic analysis suggests that the $F A F$ genes originated from a FAF-like gene and that today's $F A F$ genes arose through several rounds of duplications within the dicotyledonous plants (Additional File 1 Figure S2). FAF genes were not apparent in the rice genome or any other monocotyledonous species, even though proteins sharing homology with the Arabidopsis thaliana FAF-like gene were clearly present (Additional File 1 Table S1). Sequence homology searches failed to identify any potentially homologous proteins outside the plant kingdom, indicating that the $F A F$ gene family is plant-, possibly eudicotyledonous-specific.

\section{Expression of FAF genes throughout development}

In order to determine the temporal and spatial regulation of the expression of the four FAF genes throughout development, we consulted the AtGenExpress Arabidopsis thaliana expression atlas [19]. All four FAF transcripts were detectable throughout development (Figure 1). Expression of $F A F 1$ and $F A F 2$ at the shoot apex increased during the transition to flowering, while FAF3 and FAF4 decreased, confirming the results observed in the first microarray dataset (Additional File 1 Figure S1). However, FAF1 and FAF2 exhibited strong differences in their expression profiles in other tissues. For example, while $F A F 1$ and $F A F 2$ were both highly expressed in the apical region during the floral transition, only FAF2 expression was maintained at high levels during later stages of flower development, especially in carpels. In contrast, FAF1 expression appeared to be more transient, with some expression maintained in stamens. Similarly to $F A F 2$, FAF3 was expressed in stamens, but was also strongly expressed in the youngest leaves formed by the plant (Figure 1). This expression, however, disappeared as the leaves aged. Expression of all four FAF genes was detectable in young siliques, but expression faded as seed maturation progressed. Taken together, our analysis of microarray data showed that the FAF genes are dynamically expressed throughout development.

Table 1 Properties of Arabidopsis thaliana FAF proteins

\begin{tabular}{|c|c|c|c|c|c|c|}
\hline \multirow[b]{2}{*}{ Gene } & \multirow[b]{2}{*}{ AGI } & \multirow[b]{2}{*}{ Annotation } & \multicolumn{4}{|c|}{ Protein properties } \\
\hline & & & Length (aa) & Mass (kDa) & pl & Domains of known function \\
\hline FAF1 & At4g02810 & expressed protein & 271 & 31.2 & 4.08 & none \\
\hline FAF2 & At1g03170 & expressed protein & 240 & 27.3 & 4.74 & none \\
\hline FAF3 & At5g19260 & expressed protein & 288 & 32.1 & 4.34 & none \\
\hline FAF4 & At3g06020 & expressed protein & 300 & 33.9 & 4.88 & none \\
\hline
\end{tabular}




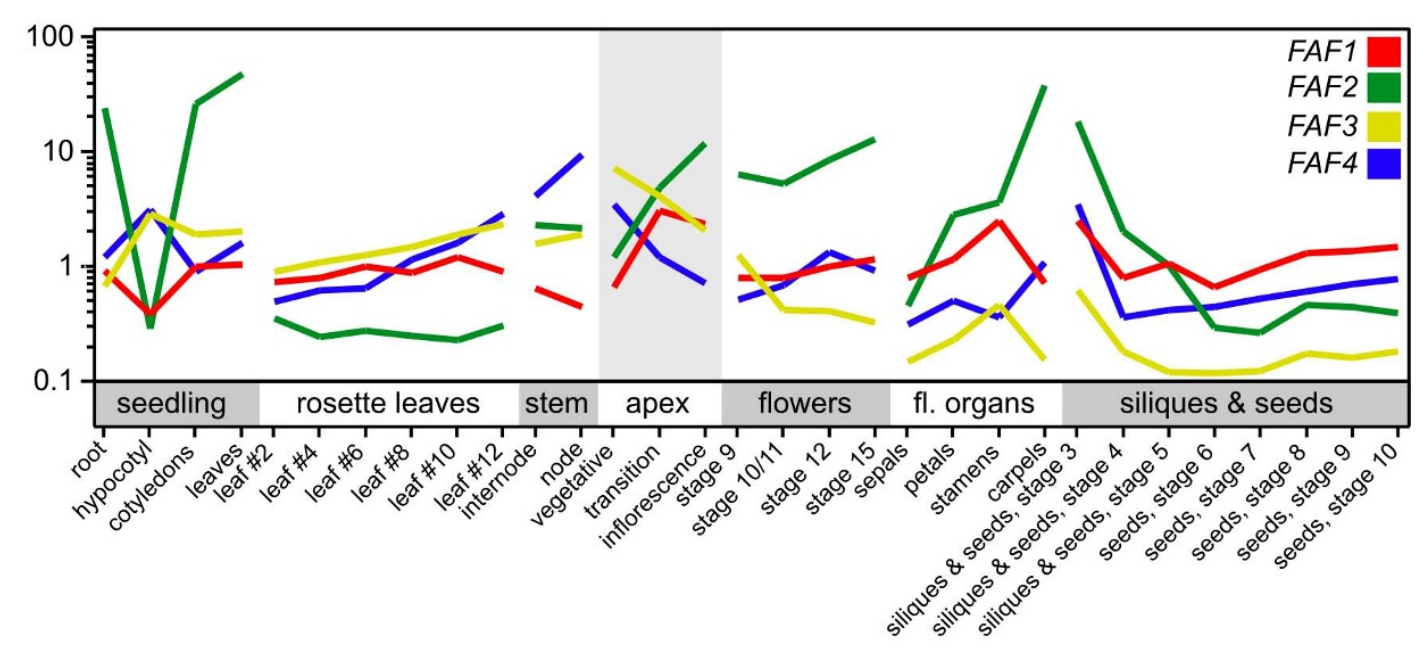

Figure 1 Microarray expression profiles of the FAF gene family. Expression of FAF genes in selected tissues from the 'AtGenExpress' expression atlas of Arabidopsis thaliana development. Samples confirming the expression changes observed at the apex during the floral transition (Additional File 1 Figure S1) are shaded.

\section{FAF genes are expressed in the centre of the shoot meristem and in vascular tissue}

To analyze FAF expression at cellular resolution, we carried out RNA in situ hybridization (Figure 2). Expression of all four FAF genes was detected in provascular and vascular tissue at different stages throughout development. FAF1 and FAF2 were only weakly expressed in the vasculature of vegetative plants (Figure $2 \mathrm{~B}, \mathrm{C}, \mathrm{G}, \mathrm{H}$ ). In addition to the vasculature, FAF2 mRNA was also detectable in the centre of the vegetative meristem (Figure $2 \mathrm{C}$ ). In contrast, FAF3 and FAF4 could easily be detected in the vasculature (Figure 2D, E, I, J; arrows), but neither seemed to be expressed in the vegetative meristem (Figure 2D, E).

Expression of the FAF genes changed upon the onset of flowering (Figure 2L-O), as already observed in the microarray experiments (Figure 1). FAF1 and FAF2 were induced in the inflorescence vasculature and young flower buds as flowering commenced (Figure 2L, M). In contrast, FAF3 and FAF4 expression in inflorescences was restricted to the vasculature, but was largely absent from young flowers (Figure 2N, O). Both, FAF2 and FAF4 were, however, detected in the centre of the inflorescence meristem (Figure $2 \mathrm{M}, \mathrm{O}$; arrowheads).

Upon fertilization, expression of FAF1, FAF3, FAF4, but not $F A F 2$ could also be detected in the developing embryo, starting from the early heart stage and lasting until torpedo stage (Figure 2Q-T). FAF2 expression was, however, detectable in the funiculus (Figure $2 \mathrm{~V}$, arrowhead).

The dynamic nature of $F A F$ gene regulation was confirmed by the dramatic changes in reporter gene activity observed during the first 8 days after germination
(Additional File 1 Figure S3). FAF1::GUS activity, for example, was initially restricted to the hypocotyl, but expression gradually shifted to the root over the following four days. Starting on day 6, FAF1::GUS became active in the vasculature of the cotyledons and subsequently also in the leaves. Similar, but distinct, dynamic regulation of reporter gene activity could also be observed for the other $F A F$ promoters (Additional File 1 Figure S3). In addition, FAF2::GUS was observed in the centre of the vegetative shoot meristem (Additional File 1 Figure $\mathrm{S} 4 \mathrm{H}$ ) as already shown by RNA in situ hybridization (Figure 2M). After the onset of flowering, FAF1:: GUS was observed most strongly in anthers (Additional File 1 Figure S4A), while FAF2::GUS expression was strongest in the carpel, particularly in the funiculus (Additional File 1 Figure S4B, G), where FAF2 RNA had also been detected (Figure 2V). FAF3::GUS activity was restricted to anthers (Additional File 1 Figure S4C), whereas FAF4 was expressed at the base of the flower and in the vasculature of the pedicels and the inflorescence stem (Additional File 1 Figure S4D). In differentiated tissues such as root and leaves, the $F A F$ genes were predominantly expressed in the phloem, as shown for FAF2 (Additional File 1 Figure S3E, F).

In summary, FAF2 and FAF4 are expressed in the centre of the shoot meristem, suggesting a potential role for these two FAF proteins in meristem development. In addition, all $F A F$ genes are expressed in the vasculature, where they may function in a partially redundant manner.

\section{FAF proteins can affect growth and meristem size}

To study the function of the FAF proteins during development, we first searched for knock-out lines (Additional 

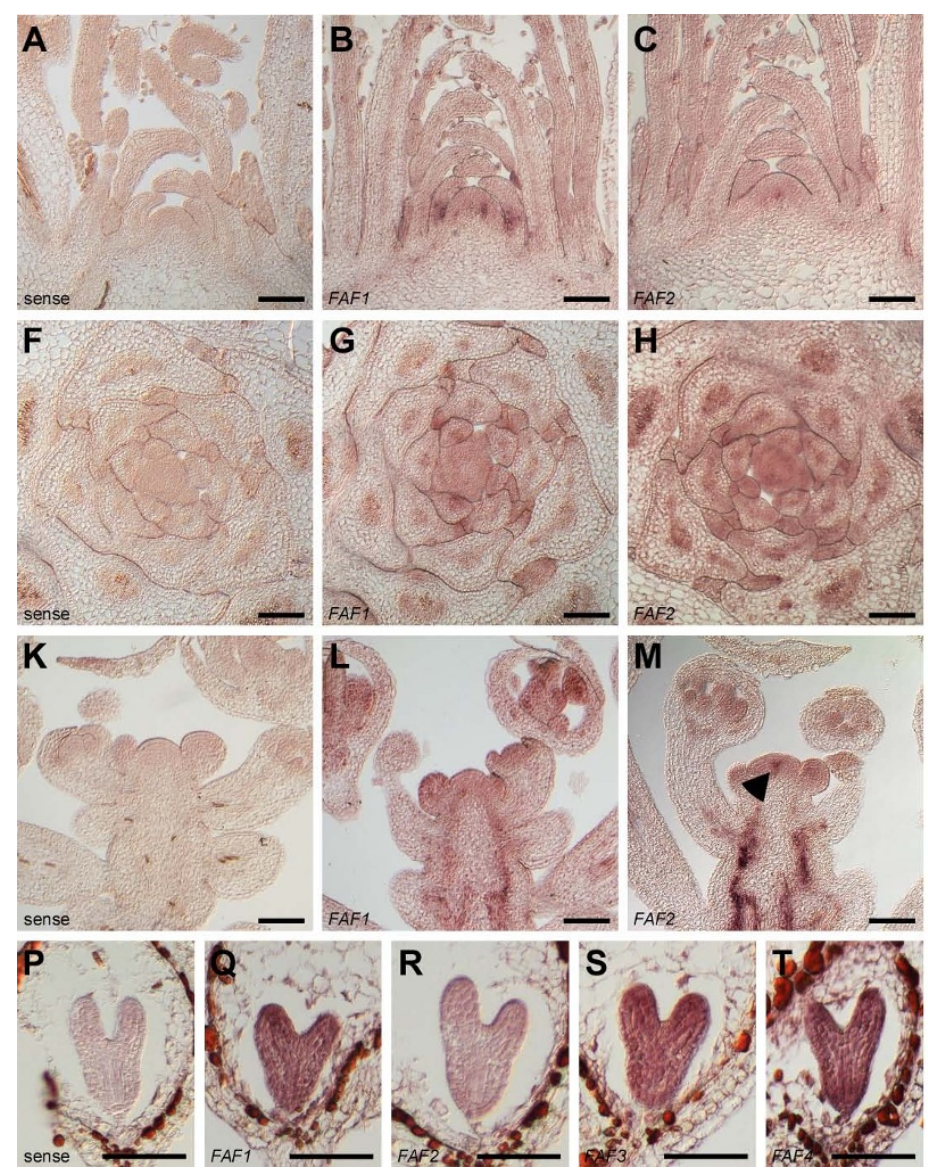
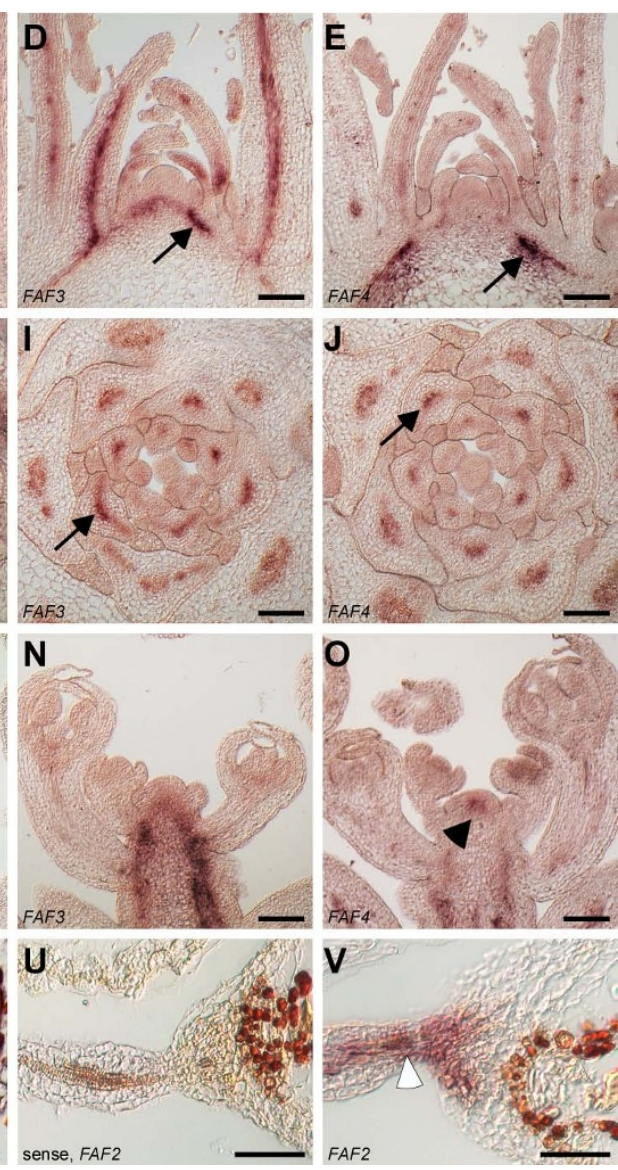

Figure 2 Expression patterns of the FAF genes throughout development assayed by RNA in situ hybridization. (A-J) Expression of the FAF genes at the vegetative apex. Longitudinal $(\mathbf{A}-\mathbf{E})$ and transverse sections $(\mathbf{F}-\mathbf{J})$ through the vegetative apex hybridized with sense $(\mathbf{A}, \mathbf{F})$ and antisense probes (B-E, G-J) against the four FAF genes are shown. Highest expression was detected for FAF3 and FAF4 in the vascular and provascular tissue (D, E, I, J, arrows). (K-O) In inflorescences, FAF1 expression (L) was detected in the developing vasculature and young flowers. FAF2 expression (M) was highest in the inflorescence stem, but also detectable in the centre of the meristem (M, arrowhead). Expression of FAF3 was restricted to the developing vasculature (N), while FAF4 was also found in the centre of the meristem (O, arrowhead). No signal was found when sense probes were used (K). (P-V) During embryogenesis, FAF7 (Q), FAF3 (S), and FAF4 (T) were expressed in the embryo from heart stage onward, while expression of FAF2 was limited to the funiculus (V). Sense probes (P, U) did not result in any staining. Scale bars: $100 \mu \mathrm{m}(\mathrm{A}-\mathrm{O})$, $50 \mu \mathrm{m}$ (P-T).

File 1 Table S2). Most of the lines investigated showed either wild-type mRNA levels, indicating that expression of the corresponding FAF gene was unaltered in these lines or the presence of the T-DNA could not be confirmed or the lines were not available from the stock centre. Only for FAF3 a potential RNA-null line (SM_3_40331) could be recovered. This line, however, did not show an obvious phenotype, possibly due to redundancy with the other $F A F$ genes. Attempts to knock-down individual or certain combinations of $F A F$ genes by constitutive and inducible RNAi (Additional File 1 Table S3) resulted in pleiotropic phenotypes in all $\mathrm{T} 1$ lines investigated. Unfortunately, all lines that eventually did set seeds were silenced in T2, making further analysis impracticable. Besides regular RNAi, artificial microRNAs (Additional File 1 Table S4) were prepared to knock-down FAF
mRNAs either individually or in combination, but these did not result in a significant degradation of the targeted transcripts and lines showed no discernable phenotypes $[20,21]$. Finally, tilling of $F A F$ genes (Additional File 1 Table S5) also failed to produce alleles with major changes such as premature stop codons [22,23].

Given the difficulty of obtaining loss-of-function lines, we resorted to misexpression experiments. We constitutively expressed $F A F$ genes under the control of the viral $35 S$ promoter in planta. In general we observed similar phenotypes, regardless of which $F A F$ gene was overexpressed, indicating that all four FAF proteins can perform the same function. Lines expressing $F A F$ genes at a very high level, as determined by qRT-PCR (data not shown), arrested shoot growth shortly after germination (Figure 3A, B). Arrest this early in development 

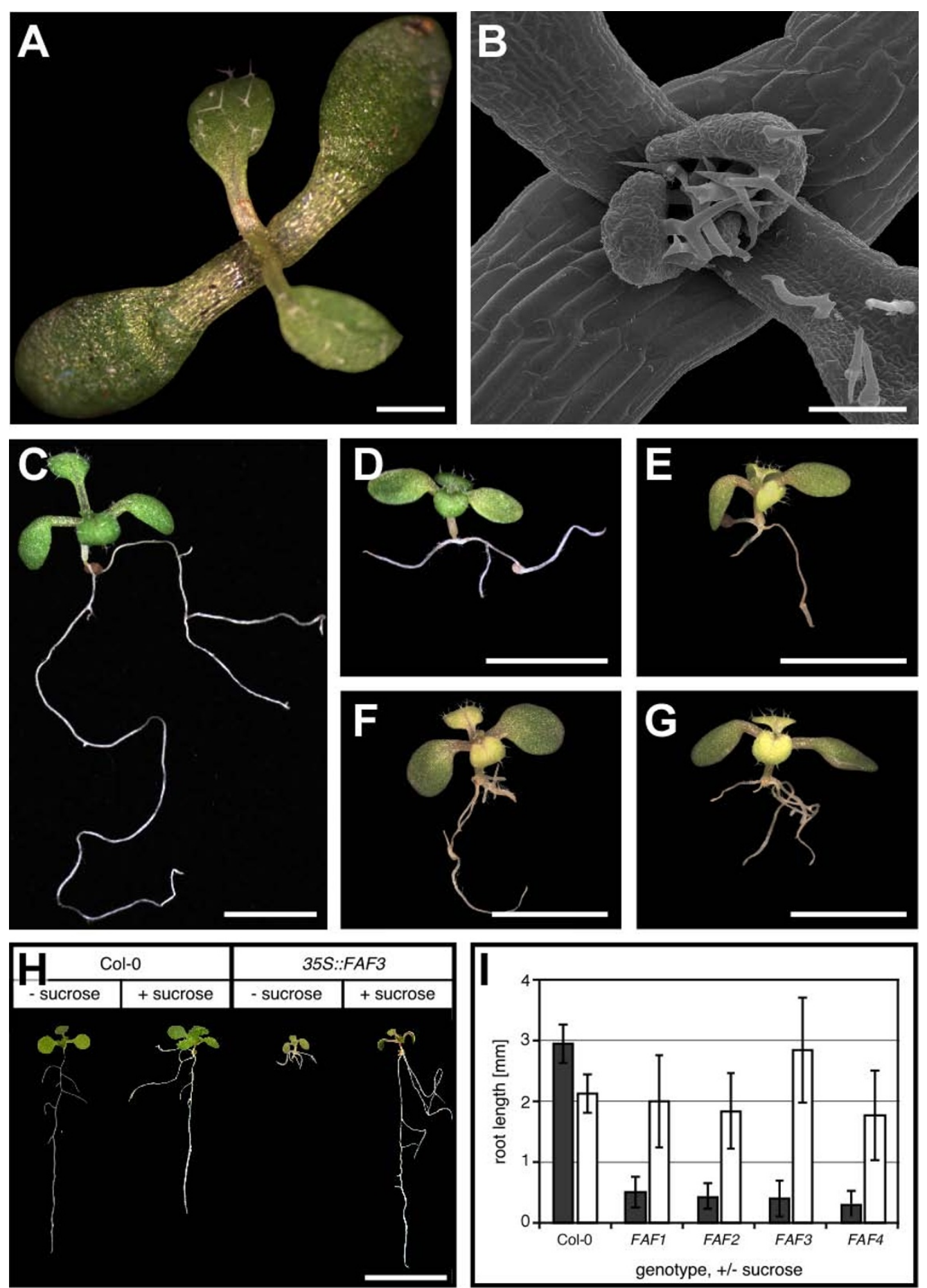

Figure 3 Arrest of shoot and root growth by constitutive FAF expression. (A) Arrested shoot meristem in a strong 35S.:FAF3 seedling Expression of the other FAF genes by the $35 \mathrm{~S}$ promoter caused similar phenotypes (data not shown) (B) Close-up of arrested seedling under the SEM. (C-G) Root development of wild-type control (C) and intermediate 355::FAF1 (D), 355::FAF2 (E), 35S::FAF3 (F), and 35S::FAF4 (G) plants. The growth of the primary root is inhibited and the formation of adventitious roots is induced by high levels of FAF expression (D-G). (H) Rescue of root growth of a 355::FAF3 line by exogenous sucrose (1\%). (I) Quantification of the effect of sucrose on root growth in Col-0 and 355::FAF plants $(n=20)$. Scale bars: $0.5 \mathrm{~mm}(A), 200 \mu \mathrm{m}(B), 5 \mathrm{~mm}(C), 2 \mathrm{~mm}(\mathrm{D}-\mathrm{G}), 1 \mathrm{~cm}$. (H). 
was observed in $2 \%$ (FAF2) to $12 \%$ (FAF3) of independent $\mathrm{T} 1$ lines ( $>140$ per $F A F$ gene).

The strongest lines were sterile, therefore we focused our analysis on those plants with intermediate expression levels ( $21 \%$ to $36 \%$ of independent $\mathrm{T} 1$ lines), for which stable lines could be established. In these lines we observed a strong reduction in root growth (Figure 3DG) when compared to wild-type plants (Figure 3C). This was accompanied by an increased formation of adventitious roots at the hypocotyl. The arrest of the root growth could be overcome when $1 \%$ sucrose was supplied in the medium (Figure $3 \mathrm{H}, \mathrm{I}$ ).

Moderate $F A F$ overexpressing plants were smaller than wild-type, and leaf vasculature appeared to be reduced (not shown). Apart from this they developed normally, until after the transition to flowering and bolting, at which point inflorescence meristems ceased producing new organs and shoot elongation stopped (Figure 4A and inset). In the last flowers to be formed before the meristem arrested, floral organs, in particular the stamens and carpel, were retarded in development (Figure 4A, inset). When we examined the meristems in more detail (Figure $4 B, C$ ), we found that the width of the inflorescence meristems in FAF overexpressing lines was on average reduced by approximately $30 \%$ when compared to wild-type (Figure 4D).

\section{FAF proteins can repress WUSCHEL in the organizing centre of the shoot meristem}

Loss of WUS function results in a reduction of meristem size, similar to what we observed in FAF overexpressing lines. Moreover, two FAF genes are expressed in the centre of the meristem, overlapping with the site of WUS expression in the OC. This prompted us to analyze expression of WUS in the meristem of FAF overexpressing lines (Figure $5 \mathrm{~A}-\mathrm{E}$ ). We found that WUS expression was strongly reduced in both inflorescence and flower meristems. Since WUS is required for maintenance of meristem function, the reduction in WUS expression is consistent with the meristem arrest phenotype seen in strong (Figure 3A) and moderate (Figure 4) $F A F$ overexpressing plants.

Expression of WUS in the OC of the shoot meristem is under negative control of CLV3-dependent signalling. We found that $C L V 3$ expression was essentially normal in $F A F$ overexpressing lines (Figure $5 \mathrm{~F}-\mathrm{J}$ ), indicating that the reduction in WUS expression was not caused by an increase or expansion of CLV3 expression.

\section{Repression of FAF2 and FAF4 in the shoot meristem by CLAVATA3}

The fact that WUS expression is reduced in FAF overexpressing lines suggested that FAF2 and FAF4, which are normally expressed in the meristem, might be involved in the CLV3 mediated repression of WUS. We therefore analyzed FAF2 and FAF4 expression in $c l v 3-7$ mutants (Figure 6). We found that expression of FAF2 was strongly enhanced in the centre of $c l v 3-7$ inflorescence meristems (Figure 6A, C), while its expression in the vasculature appeared to be not affected. Although meristems are enlarged in clv3-7 mutants, the simple increase in cell number does not explain the strong staining observed, suggesting that FAF2 is under repression by CLV3. Similarly, we found FAF4 to be expressed more strongly in the enlarged centre of clv3-7 meristems (Figure 6B, D), though the increase was not as pronounced as for $F A F 2$. In order to confirm the upregulation of FAF2 and FAF4 in the inflorescence meristem of clv3-7 mutants, we analyzed microarray expression data of Col-0 and clv3-7 inflorescence meristems from the AtGenExpress transcriptome atlas. We found significant $(\operatorname{logitT} \mathrm{p}<0.01)$ and strong induction of FAF2 (2.2-fold) and FAF4 (2.5-fold) in clv3-7 inflorescence meristems when compared to Col-0 control plants (Figure 6E). Confirming the quality of the array data, WUS was also found to be significantly and strongly (2.9-fold) induced in the clv3-7 mutant. Neither FAF1 nor FAF3 changed significantly and strongly (> 2-fold) in the $\operatorname{clv} 3-7$ microarray data set.

The observed upregulation of FAF2 and FAF4 in clv37 inflorescence meristems could either indicate that these two FAF genes are under repression by CLV3 or that they are positively regulated by WUS. To be able to distinguish between these two possibilities we examined the response of FAF genes to inducible ectopic WUS expression in a microarray dataset from 12-day-old seedlings [24]. We found that none of the FAF genes were induced, suggesting that they are not positively regulated by WUS but are more likely to be under repression by CLV3 (Figure 6F).

Taken together, our results indicate that FAF proteins, when expressed at high levels, can affect shoot meristem size in Arabidopsis thaliana by modulating CLV3dependent WUS expression. In wild-type plants, only FAF2 and FAF4 are likely to participate in the regulation of WUS since only these two genes are normally expressed in the centre of the shoot meristem. In addition, FAF2 and FAF4 expression in the meristem appears to be under negative control by the CLV3. However, the observation that constitutive expression of any of the four FAFs can affect meristem size demonstrates that the ability to repress WUS is intrinsic to all four FAF proteins.

\section{Discussion}

The shoot apical meristem is initiated early during embryogenesis and harbours a small population of pluripotent stem cells from which all aerial parts of the 


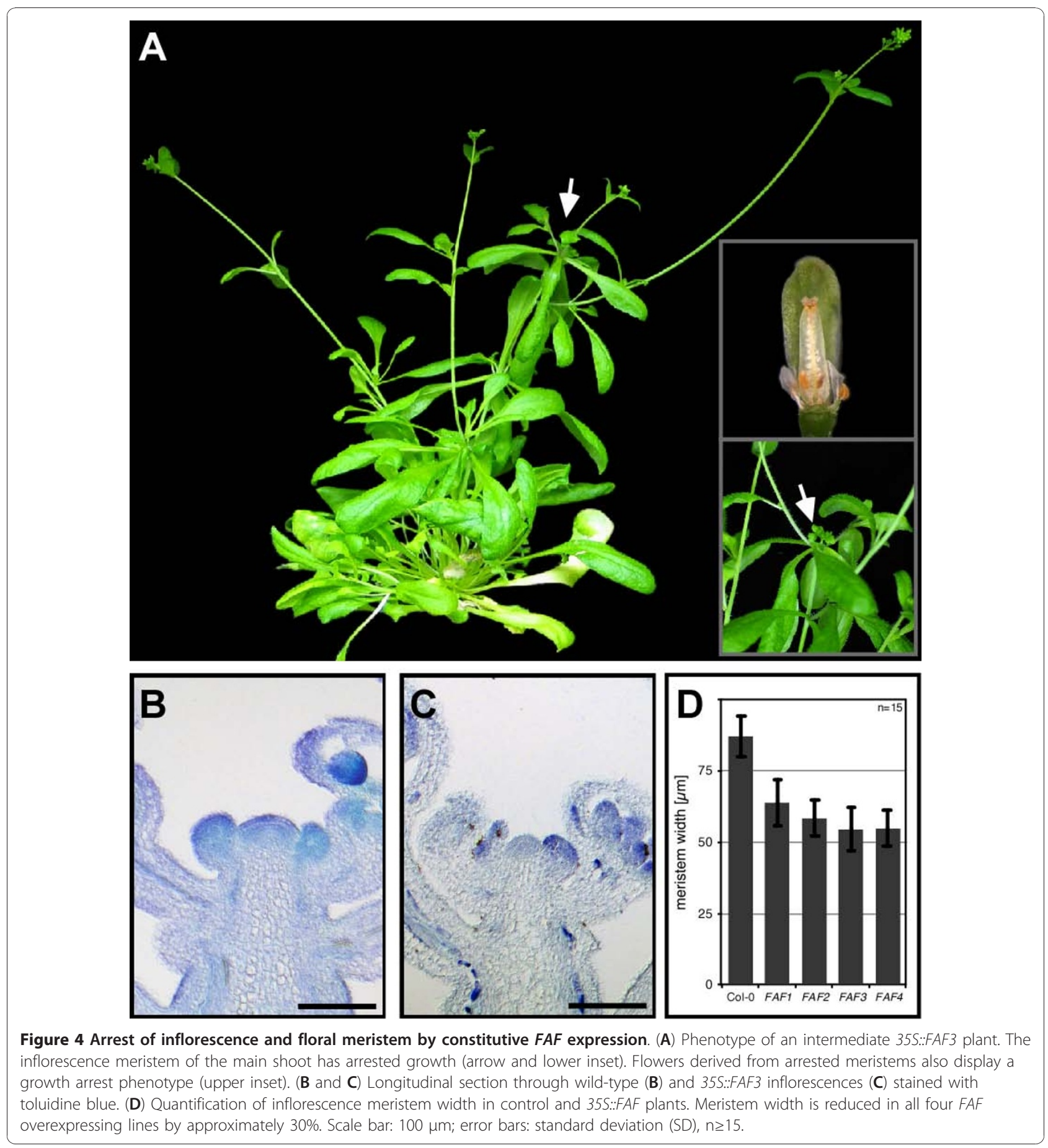

plant are derived [1,25]. Establishment and maintenance of these stem cells depends on the activity of the WUS and $C L V$ genes, which are mutually regulating each other's expression in a spatial negative feedback loop [3]. WUS expression in the $\mathrm{OC}$ of the shoot meristem promotes stem cell fate in the cells above while the stem cells themselves secrete a small peptide, CLV3, which is perceived by CLV1 and, possibly, the CLV2/ CRN receptor complex $[3,11,12,26]$. Ultimately, CLV3dependent signalling limits the size of the WUS-expressing OC. The WUS-CLV system is rather dynamic and can, over time, compensate for even 10-fold differences in $C L V 3$ expression, indicating that $C L V 3$ expression confers information about stem-cell position to the 

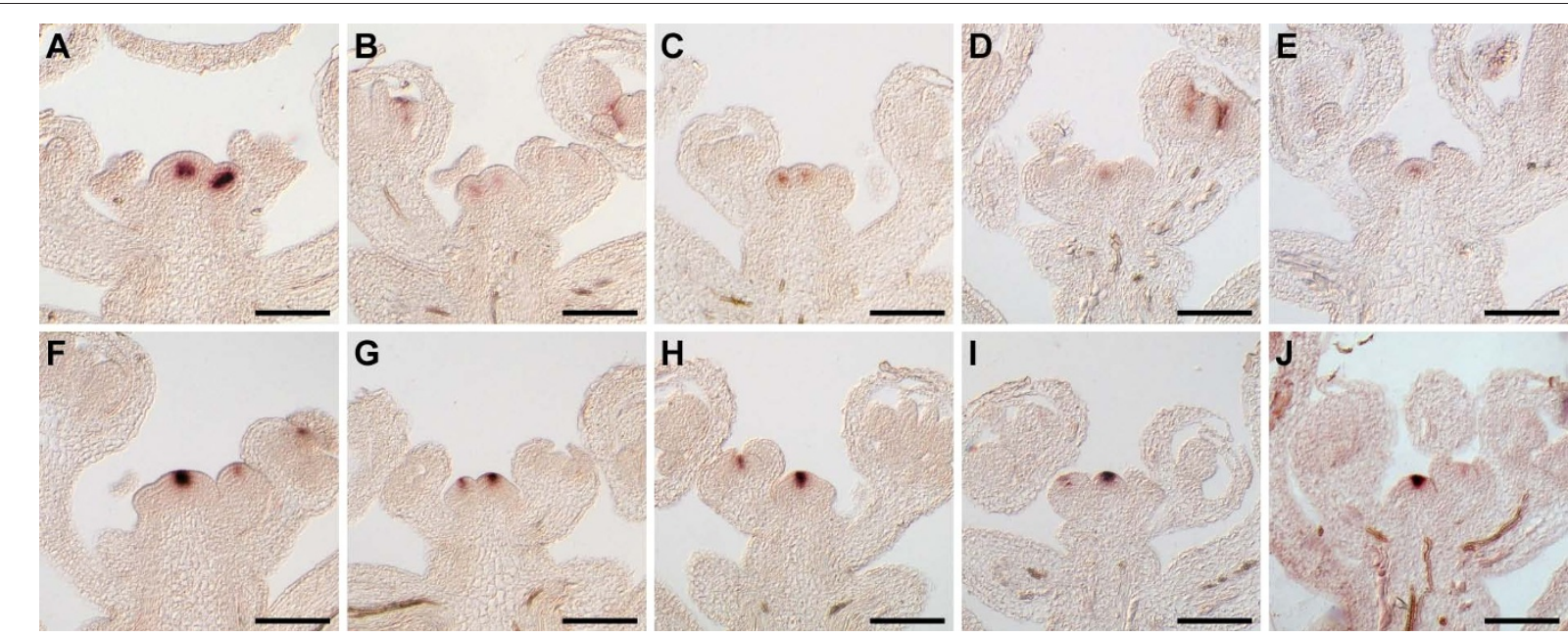

Figure 5 Effect of FAF genes on WUS and CLV3 gene expression. Detection of WUS (A-E) and CLV3 (F-J) transcripts by RNA in situ hybridization in wild-type (A, F), 35S::FAF1 (B, G), 355::FAF2 (C, H), 355::FAF3 (D, I), and 355.:.FAF4 (E, J). WUS expression is reduced (B-E) while CLV3 expression (G-J) appears normal in 35S:.:FAF plants. Scale bar: $100 \mu \mathrm{m}$.

underlying $\mathrm{OC}$ rather than information about stem cell number [14].

Analysis of FAF overexpressing lines by RNA in situ hybridization demonstrated that WUS was strongly downregulated in these lines. The fact that the expression of WUS was affected regardless of which FAF gene was constitutively expressed, suggests that the ability to repress WUS is intrinsic to all four FAF proteins. In wild-type, $F A F$ effects on WUS are likely to be exerted only by FAF2 and FAF4, which are the two FAF genes expressed in the centre of the shoot and/or inflorescence meristem in a domain that appears to be overlapping with the site of WUS expression.

In the clv3-7 mutant the expression domains of WUS and FAF2/FAF4 appear to be largely exclusive. WUS is limited to the second meristem layer (L2) but is no longer detectable in the centre of the meristem $[7,27]$. In contrast, expression of $F A F 2$ and FAF4 were found to be upregulated in the centre of the meristem but are mostly excluded from the L2. This suggests that in wildtype expression of FAF2/FAF4 might attenuate WUS expression in the centre of the meristem whereas high levels of FAF2/FAF4 in clv3-7 prevent WUS from being expressed in the centre of the meristem and limit its expression to the L2. Based on our results, we propose that $F A F$ genes function in the shoot meristem, with CLV3 negatively regulating FAF2 and FAF4 expression, which in turn contribute to the repression of WUS. In this context it is interesting to note that all four FAF proteins harbour a short sequence motif (L-X-L-X-L) that is reminiscent of the EAR repression motif [28]. This would be in agreement with the proposed role of FAF proteins as repressors of WUS.
Expression of FAF2 and FAF4 in the centre of the meristem would put them in place to compensate for the effects of positive regulators such as STIMPY on WUS expression in the OC. Interestingly, we found that CLV3 expression was not decreased in FAF overexpression lines, even though WUS levels were severely reduced. Expression of WUS in the OC is under constant surveillance by several other positive and negative regulators [reviewed in 1, 29]. For example, in $j b a-1 D$ plants, a mutant in which the miR166g is overexpressed, WUS expression is highly induced, while the relative level of CLV3 transcription remains unchanged compared with wild-type plants [30]. These observations together with data presented here suggest that the expression of $C L V 3$ is maintained over a wide range of WUS levels, similar to what has been shown for the effect of CLV3 on WUS [14]. In addition, several other transcription factors, as well as a number of proteins involved in chromatin remodelling, have been shown to regulate WUS. Having established the FAF proteins as negative regulators of WUS, it will be interesting to analyze possible genetic interactions between the $F A F$ genes and the other WUS regulators in detail.

WUS is not only expressed in the OC of the shoot meristem, but also in young flower meristems, where it directly regulates expression of the homeotic gene $A G A$ MOUS (AG) in the centre of the newly formed flower [31,32]. AG is normally required for the development of the inner two whorls of the flower [33]. Reduction of WUS expression in the flower meristem could result in a downregulation of $A G$, which could explain the observed defects in flowers of $F A F$ overexpressing plants. 


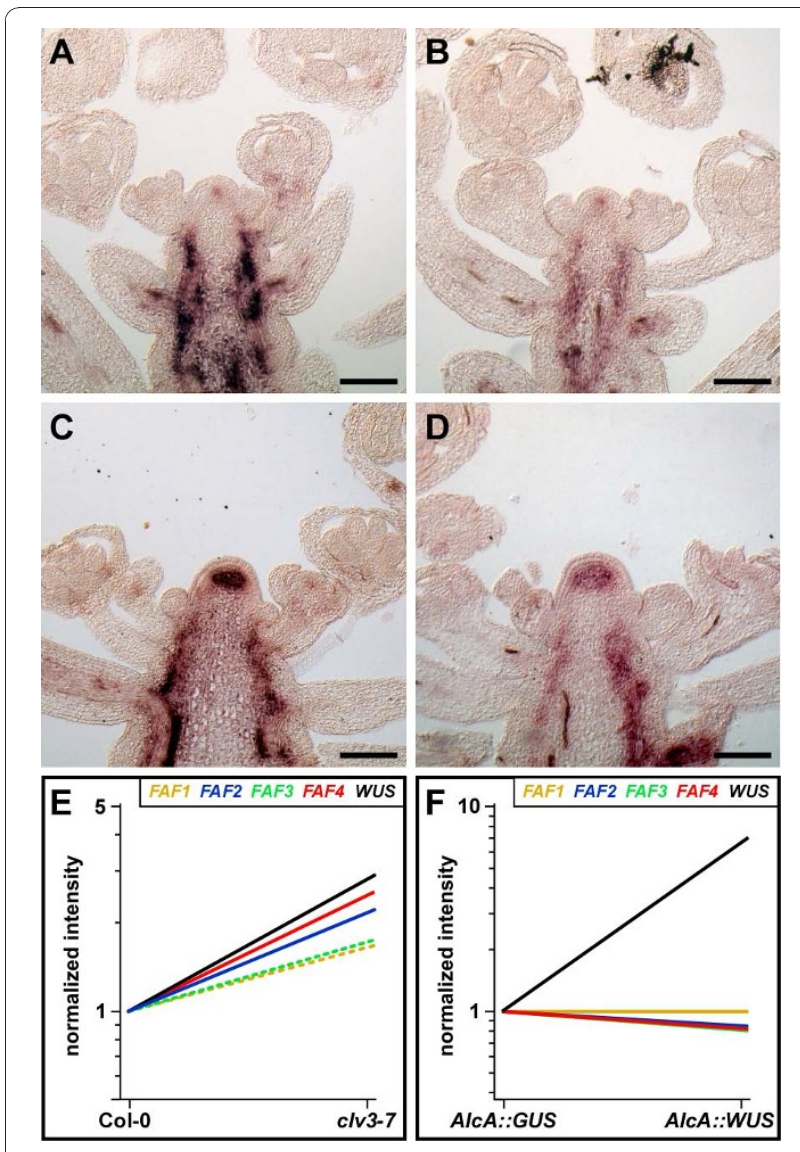

Figure 6 Negative regulation of FAF2 and FAF4 expression in the organizing centre of the shoot meristem by CLV3.

Expression of FAF2 $(\mathbf{A}, \mathbf{C})$ and FAF4 (B, D) in wild-type control plants $(\mathbf{A}, \mathbf{B})$ and clv3-7 mutants (C, D). Expression of FAF2 (C) and FAF4 (D) is elevated in clv3-7 mutants when compared to wild-type controls (A, B). (E, F) Microarray expression profiles of WUS and the FAF genes. (E) WUS, FAF2, and FAF4 are significantly upregulated and change more than 2-fold (solid lines) in clv3-7, while FAF1 and FAF3 do not (dashed lines). (F) FAF genes do not respond to ectopic WUS expression. Scale bar: $100 \mu \mathrm{m}$.

Apart from defects in the shoot meristem, FAF overexpression resulted in an arrested root meristem. This finding suggests that the FAF proteins can influence meristem maintenance at both poles of the growing plant. Since WUS is not expressed in the root meristem, it will be interesting to investigate, which $W O X$ gene takes on its function in the root. STIMPY (STIP; WOX9), a homeodomain transcription factor related to WUS, has recently been shown to promote WUS expression in the vegetative shoot meristem [16]. Based on the severity of loss-of-function alleles on both the shoot and the root meristems, STIP seems to play a more general role in meristem maintenance than WUS. In this context it is interesting to note that, similar to FAF overexpression, loss of STIP function can be compensated for by exogenous sucrose, which is in agreement with the proposed function for STIP in maintaining cell division. This suggests that STIP and the FAFs might have opposing functions in integrating sugar signalling into the meristem maintenance network.

The FAF proteins are likely to have functions other than meristem maintenance since all are expressed in vascular tissue. Consistent with a functional role for the FAFs in these tissues, we observed a reduction of tertiary and quaternary vein formation in $F A F$ overexpressing lines (data not shown). It has been reported that CLV1 and a CLV1-like gene are expressed in the phloem and cambium. Also, two members of the CLAVATA3/ESRRELATED (CLE) family, CLE6 and CLE26, are preferentially expressed in the phloem and/or the cambium [34], and it has recently been shown that application of dodecapeptides with two hydroxyproline residues encoded by the $C L E$ gene family suppress xylem cell differentiation and promote cell division in Zinnia cell cultures [35]. Thus it seems possible that FAFs affect vascular development by a mechanism similar to the one we propose for FAF function in the shoot meristem. In such a scenario the FAF proteins would act as general repressors of cell division in both the cambium and the root and shoot meristem, but are themselves under the control of the different CLAVATA/CLE proteins. Taken together our findings suggest that FAF proteins might act as transcriptional regulators, the question how exactly they exert their function remains to be determined.

\section{Conclusions}

Our study demonstrates that the four Arabidopsis thaliana FAF genes most likely arose from the FAF-like gene present in both monocotyledonous and dicotyledonous plant species, through two rounds of gene duplications. The expression of the $F A F$ genes is under developmental regulation and individual $F A F$ genes are expressed in distinct, though overlapping domains. The latter suggests that the FAF proteins might act partially redundant, which would explain why T-DNA insertion lines (as far as they could be confirmed) were indistinguishable from wild-type plants. Consistent with a certain amount of redundancy among the $F A F$ genes, RNAi and artificial microRNAs to knock-down individual or at maximum two FAF genes also did not result in any consistent and reproducible phenotypes. Based on the expression of FAF2 and FAF4 in the centre of the shoot apex, however, we assume a role of these two members of the FAF family in the shoot meristem. Supporting this idea was the finding that constitutive overexpression of the $F A F$ genes resulted in a marked reduction of meristem size. In addition, expression of WUS, a central player in the regulation of meristem size was strongly reduced in the $F A F$ misexpression lines. Finally, expression of FAF2 and FAF4 themselves appear to be under 
the control of the WUS-CLV3 feedback loop, as these two FAF genes were strongly induced in the meristem of a clv3 mutant. Taken together, our data suggest a scenario in which FAF2 and FAF4 modulate meristem size while the function of the other two FAF genes remains to be investigated.

\section{Methods}

\section{Plant material}

All lines analyzed were in the Columbia (Col-0) background. Plants were grown either under long day (LD, $16 \mathrm{~h}$ light, $8 \mathrm{~h}$ darkness) or short day (SD, 8 h light, $16 \mathrm{~h}$ darkness) conditions at $65 \%$ relative humidity under a 2:1 mixture of Cool White (Sylvania, \#0001510) and Warm White (Sylvania, \#0001511) fluorescent lights, with a fluence rate of 125 to $175 \mu \mathrm{mol} \mathrm{m}^{-2} \mathrm{~s}^{-1}$.

\section{Phylogenetic analysis}

Potential homologs of the Arabidopsis thaliana FAF and FAF-like proteins were identified by reciprocal BLAST analysis. First, we queried public databases (NCBI; Phytozome V4) using 'tblastn' and 'blastp' $(\mathrm{E}<1 \mathrm{e}-5)$ to identify potentially homologous proteins. Second, all candidates were checked against TAIR 9 protein database by 'blastp'. For this either the full length proteins (when available) or the longest peptides encoded by the various ESTs were used. Only proteins that resulted in an Arabidopsis thaliana FAF or the FAF-like protein as best hit were considered to be true FAF orthologs. For phylogenetic analysis, FAF and FAF-like proteins were preselected for maximum diversity. In particular, redundant sequences from the same or closely related species were not considered and only one representative protein sequence was included in the final tree. Peptides deduced from ESTs were only considered if they completely covered the conserved domains that were eventually used to construct the phylogeny. The only exception to this was a sequence originating from Selaginella moellendorffii (Phytozome-Id: 418746) that serves as an outgroup, which contains only one of the two regions that are conserved in all FAF and FAF-like proteins. Finally, the homologs of FAF proteins were aligned with T-COFFEE [36], then only the conserved domains were used for phylogenetic analysis. PAUP* version 4.0b10 [37] was used to reconstruct the phylogenetic tree using the Neighbor-joining (NJ) method. Topological robustness was assessed by bootstrap analysis with 1000 replicates using simple taxon addition [38].

\section{Analysis of microarray expression data}

Microarray data were imported into the GeneSpring 7 software (Agilent Technologies) and normalized using gcRMA, implemented in GeneSpring 7 [39]. Additional 'per gene' normalization was performed in GeneSpring
7. Significant changes in gene expression were calculated using logit- $\mathrm{T}$ with a cut-off of $\mathrm{p}<0.025$ [40]. Lists of differentially expressed genes were imported into GeneSpring 7 for further analysis.

\section{Molecular work and cloning}

All constructs created in this study that involved PCR were confirmed by DNA sequencing. See Additional File 1 Table $\mathrm{S} 6$ for information on the sequences of the oligonucleotides used. All four $F A F$ genes are encoded by single exon genes. For the construction of overexpressing lines, protein coding region were amplified from genomic DNA and cloned into the pCRsmart vector, a derivative of pBluescript. ORFs were than cloned as BamHI-Pst I fragments into the shuttle vector pBJ36-35 S. Cassettes containing the $35 S$ promoter, the FAF ORF and the ocs terminator were excised from the respective plasmids using NotI, ligated into the pMLBART binary vector and transformed into Col0wild-type plants by floral dipping [41]. For the $\beta$-glucuronidase (GUS) reporters, $2.5 \mathrm{~kb}$ fragments upstream of the FAF start codon were amplified by PCR, cloned into the vector pRITA, which contains the GUS gene followed by a nos terminator. The entire cassettes were excised with NotI and ligated into the pMLBART binary vector that provides resistance to the herbicide glufosinate (Basta, Bayer CropScience) in plants.

\section{Scanning electron microscopy (SEM)}

Tissue was fixed for 5 minutes in 100\% methanol, followed by 3-5 washes with $100 \%$ ethanol. Further preparation was carried out as described [42]. Images were acquired on a Hitachi S800 electron microscope, at an accelerating voltage of $20 \mathrm{kV}$.

\section{RNA in situ hybridization and GUS staining}

RNA in situ hybridization was performed largely as previously described [42], but infiltration with paraffin was carried out using an ASP300 automated embedding apparatus (Leica). Sections $(9-12 \mu \mathrm{m})$ were prepared with an EG1160 microtome (Leica). Sense probes were tested for all genes, but did not result in any noticeable staining and were therefore omitted from most figures. Sections shown in different panels in a given figure were processed in parallel and the signal was allowed to develop for the same time to ensure comparability. Images were taken on an Axioplan2 microscope (Zeiss) equipped with an AxioCam HRc (Zeiss) digital camera. GUS staining was carried out as described [42]. Whole mount preparations were examined under an MZ FLIII (Leica) microscope and pictures were taken with an AxioCam HRc digital camera (Zeiss). Thin sections of tissues stained for GUS activity were prepared from paraffin embedded tissue as described above. 
The width of the inflorescence meristem was determined on tissue sections stained with toluidine blue. For this purpose, serial sections of the meristem were prepared and the width of the meristem was determined from the section that passed through the centre of the meristem. The average meristem width and the standard deviation were calculated based on measurements of 15 meristems.

\section{Additional material}

Additional file 1: • Table S1. FAF-like proteins from Arabidopsis thaliana and several monocotyledonous species. - Table S2. FAF T-DNA insertion lines in Col-0 background. • Table S3. FAF RNAi hair-pin constructs. • Table S4. Artificial miRNAs targeting FAF transcripts. • Table S5. Summary of FAF tilling lines. - Table S6. Oligonucleotides used in this study. • Figure S1. Expression profiles of FAF genes in response to long day. • Figure S2. Phylogenetic analysis of the plant-specific FAF protein family. • Figure S3. GUS expression in seedlings of FAF reporter lines. - Figure S4. GUS reporter activity in the meristem and reproductive organs.

\section{Acknowledgements}

The authors would like to thank Sarah N. Fehr, Tanja Weinand, and David S. M. Antonio for help with plant work and Jürgen Berger for skillful assistance with scanning electron microscopy. We also thank Detlef Weigel, Jan Lohmann, Vojislava Grbic, Kirsten Bomblies, John E. Lunn and Stéphanie Arrivault for many valuable comments on the manuscript. This work was supported by two grants from the Deutsche Forschungsgemeinschaft (DFG) to M.S. (SCHM 1560/4-1; SCHM 1560/6-1).

\section{Author details}

${ }^{1}$ Max Planck Institute of Molecular Plant Physiology, D-14476 Potsdam, Germany. ${ }^{2} Z$ entrum für Molekularbiologie der Pflanzen - Pflanzenphysiologie, Universität Tübingen, Auf der Morgenstelle 1, D-72076 Tübingen, Germany. ${ }^{3}$ Department of Molecular Biology, AG Schmid, Max Planck Institute for Developmental Biology, D-72076 Tübingen, Germany.

\section{Authors' contributions}

WW and MS conceived and designed the experiments. WW performed all the experiments, except for some in situ hybridizations and the phylogenetic analysis, which were carried out by LHB and YG, respectively. WW and MS analyzed the data. WW and MS wrote the paper. All authors read and approved the final manuscript.

Received: 3 August 2010 Accepted: 22 December 2010 Published: 22 December 2010

\section{References}

1. Williams L, Fletcher JC: Stem cell regulation in the Arabidopsis shoot apical meristem. Curr Opin Plant Biol 2005, 8(6):582-586.

2. Wolters $H$, Jurgens $G$ : Survival of the flexible: hormonal growth control and adaptation in plant development. Nature reviews 2009, 10(5):305-317

3. Schoof H, Lenhard M, Haecker A, Mayer KF, Jurgens G, Laux T: The stem cell population of Arabidopsis shoot meristems in maintained by a regulatory loop between the CLAVATA and WUSCHEL genes. Cell 2000, 100(6):635-644.

4. Mayer KF, Schoof H, Haecker A, Lenhard M, Jurgens G, Laux T: Role of WUSCHEL in regulating stem cell fate in the Arabidopsis shoot meristem. Cell 1998, 95(6):805-815.

5. Clark SE, Jacobsen SE, Levin JZ, Meyerowitz EM: The CLAVATA and SHOOT MERISTEMLESS loci competitively regulate meristem activity in Arabidopsis. Development 1996, 122(5):1567-1575.

6. Fletcher JC, Brand U, Running MP, Simon R, Meyerowitz EM: Signaling of cell fate decisions by CLAVATA3 in Arabidopsis shoot meristems. Science 1999, 283(5409):1911-1914
7. Brand U, Fletcher JC, Hobe M, Meyerowitz EM, Simon R: Dependence of stem cell fate in Arabidopsis on a feedback loop regulated by CLV3 activity. Science 2000, 289(5479):617-619.

8. Lenhard M, Laux T: Stem cell homeostasis in the Arabidopsis shoot meristem is regulated by intercellular movement of CLAVATA3 and its sequestration by CLAVATA1. Development 2003, 130(14):3163-3173.

9. Kondo T, Sawa S, Kinoshita A, Mizuno S, Kakimoto T, Fukuda H, Sakagami Y: A plant peptide encoded by CLV3 identified by in situ MALDI-TOF MS analysis. Science 2006, 313(5788):845-848.

10. Trotochaud AE, Hao T, Wu G, Yang Z, Clark SE: The CLAVATA1 receptorlike kinase requires CLAVATA3 for its assembly into a signaling complex that includes KAPP and a Rho-related protein. Plant Cell 1999, 11(3):393-406.

11. Ogawa M, Shinohara H, Sakagami Y, Matsubayashi Y: Arabidopsis CLV3 peptide directly binds CLV1 ectodomain. Science 2008, 319(5861):294.

12. Muller R, Bleckmann A, Simon R: The receptor kinase CORYNE of Arabidopsis transmits the stem cell-limiting signal CLAVATA3 independently of CLAVATA1. Plant Cell 2008, 20(4):934-946.

13. Miwa H, Betsuyaku S, Iwamoto K, Kinoshita A, Fukuda H, Sawa S: The receptor-like kinase SOL2 mediates CLE signaling in Arabidopsis. Plant \& cell physiology 2008, 49(11):1752-1757.

14. Muller R, Borghi L, Kwiatkowska D, Laufs P, Simon R: Dynamic and compensatory responses of Arabidopsis shoot and floral meristems to CLV3 signaling. Plant Cell 2006, 18(5):1188-1198.

15. Francis D, Halford NG: Nutrient sensing in plant meristems. Plant Mol Biol 2006, 60(6):981-993.

16. Wu X, Dabi T, Weigel D: Requirement of homeobox gene STIMPY/WOX9 for Arabidopsis meristem growth and maintenance. Curr Biol 2005, 15(5):436-440.

17. Schmid M, Uhlenhaut NH, Godard F, Demar M, Bressan R, Weigel D, Lohmann JU: Dissection of floral induction pathways using global expression analysis. Development 2003, 130(24):6001-6012.

18. Blanc $\mathrm{G}$, Wolfe $\mathrm{KH}$ : Functional divergence of duplicated genes formed by polyploidy during Arabidopsis evolution. Plant Cell 2004, 16(7):1679-1691.

19. Schmid M, Davison TS, Henz SR, Pape UJ, Demar M, Vingron M, Scholkopf B, Weigel D, Lohmann JU: A gene expression map of Arabidopsis thaliana development. Nat Genet 2005, 37(5):501-506.

20. Wesley SV, Helliwell CA, Smith NA, Wang MB, Rouse DT, Liu Q, Gooding PS, Singh SP, Abbott D, Stoutjesdijk PA, et al: Construct design for efficient, effective and high-throughput gene silencing in plants. Plant J 2001, 27(6):581-590.

21. Schwab R, Ossowski S, Riester M, Warthmann N, Weigel D: Highly specific gene silencing by artificial microRNAs in Arabidopsis. Plant Cell 2006, 18(5):1121-1133.

22. McCallum CM, Comai L, Greene EA, Henikoff S: Targeted screening for induced mutations. Nat Biotechnol 2000, 18(4):455-457.

23. McCallum CM, Comai L, Greene EA, Henikoff S: Targeting induced local lesions IN genomes (TILLING) for plant functional genomics. Plant Physiol 2000, 123(2):439-442

24. Leibfried A, To JP, Busch W, Stehling S, Kehle A, Demar M, Kieber JJ, Lohmann JU: WUSCHEL controls meristem function by direct regulation of cytokinin-inducible response regulators. Nature 2005, 438(7071):1172-1175

25. Clark SE: Cell signalling at the shoot meristem. Nat Rev Mol Cell Biol 2001, 2(4):276-284

26. Bäurle I, Laux T: Regulation of WUSCHEL transcription in the stem cell niche of the Arabidopsis shoot meristem. Plant Cell 2005, 17(8):2271-2280.

27. Busch W, Miotk A, Ariel FD, Zhao Z, Forner J, Daum G, Suzaki T, Schuster C, Schultheiss SJ, Leibfried A, et al: Transcriptional control of a plant stem cell niche. Developmental cell 2010, 18(5):849-861.

28. Ikeda M, Ohme-Takagi M: A novel group of transcriptional repressors in Arabidopsis. Plant \& cell physiology 2009, 50(5):970-975.

29. Sablowski R: The dynamic plant stem cell niches. Curr Opin Plant Biol 2007, 10(6):639-644.

30. Williams L, Grigg SP, Xie M, Christensen S, Fletcher JC: Regulation of Arabidopsis shoot apical meristem and lateral organ formation by microRNA miR166g and its AtHD-ZIP target genes. Development 2005, 132(16):3657-3668

31. Lohmann JU, Hong RL, Hobe M, Busch MA, Parcy F, Simon R, Weigel D: A molecular link between stem cell regulation and floral patterning in Arabidopsis. Cell 2001, 105(6):793-803. 
32. Lenhard M, Bohnert A, Jurgens G, Laux T: Termination of stem cell maintenance in Arabidopsis floral meristems by interactions between WUSCHEL and AGAMOUS. Cell 2001, 105(6):805-814.

33. Yanofsky MF, Ma H, Bowman JL, Drews GN, Feldmann KA, Meyerowitz EM: The protein encoded by the Arabidopsis homeotic gene agamous resembles transcription factors. Nature 1990, 346(6279):35-39.

34. Zhao C, Craig JC, Petzold HE, Dickerman AW, Beers EP: The xylem and phloem transcriptomes from secondary tissues of the Arabidopsis roothypocotyl. Plant Physiol 2005, 138(2):803-818.

35. Ito Y, Nakanomyo I, Motose H, Iwamoto K, Sawa S, Dohmae N, Fukuda H: Dodeca-CLE peptides as suppressors of plant stem cell differentiation. Science 2006, 313(5788):842-845.

36. Notredame C, Higgins DG, Heringa J: T-Coffee: A novel method for fast and accurate multiple sequence alignment. J Mol Biol 2000, 302(1):205-217.

37. Swofford DL: PAUP*. Phylogenetic Analysis Using Parsimony (*and Other Methods). Version 4 Sinauer Associates, Sunderland, Massachusetts; 2003.

38. Felsenstein J: Confidence limits on phylogenies: an approach using the bootstrap. Evolution 1985, 39(4):783-791.

39. Wu Z, Irizarry RA, Gentleman R, Martinez-Murillo F, Spencer F: A ModelBased Background Adjustment for Oligonucleotide Expression Arrays. Journal of the American Statistical Association 2004, 99(468):909-917.

40. Lemon WJ, Liyanarachchi S, You M: A high performance test of differential gene expressin for oligonucleotide arrays. Genome Biol 2003, 4:R67.

41. Gleave AP: A versatile binary vector system with a T-DNA organisational structure conducive to efficient integration of cloned DNA into the plant genome. Plant Mol Biol 1992, 20(6):1203-1207.

42. Weigel D, Glazebrook J: Arabidopsis: A laboratory manual Cold Spring Harbor, NY: Cold Spring Harbor Laboratory Press; 2002.

doi:10.1186/1471-2229-10-285

Cite this article as: Wahl et al: The FANTASTIC FOUR proteins influence shoot meristem size in Arabidopsis thaliana. BMC Plant Biology 2010 10:285.

\section{Submit your next manuscript to BioMed Central} and take full advantage of:

- Convenient online submission

- Thorough peer review

- No space constraints or color figure charges

- Immediate publication on acceptance

- Inclusion in PubMed, CAS, Scopus and Google Scholar

- Research which is freely available for redistribution 